\title{
Presencia de Toxascaris leonina y Cystoisospora felis en Lince Rojo (Lynx rufus escuinapae) en México
}

\author{
Andrea Gallardo-Santis \\ Evangelina Romero \\ Laboratorio de Diagnóstico Parasitológico, Universidad Nacional Autónoma de México, Ciudad Universitaria, 04510, \\ México, D.F. México. \\ Octavio Monroy-Vilchis \\ Martha M. Zarco-González \\ Estación Biológica Sierra Nanchititla, Facultad de Ciencias, Universidad Autónoma del Estado de México, Instituto \\ Literario 100, Centro. 50000, Toluca, México. \\ Heliot Zarza \\ Departamento de Ciencias Ambientales, CBS, Universidad Autónoma Metropolitana Unidad Lerma, Hidalgo Pte. 46, La \\ Estación, 52005, Lerma de Villada, México.h.zarza@correo.ler.uam.mx
}

El Lince Rojo (Lynx rufus) es una especie de carnívoro mediano con una amplia distribución en Norteamérica, abarcando desde el sur de Canadá hasta México (Hall 1981). En la zona central de México se encuentra la subespecie Lynx rufus escuinapae, la cual habita principalmente el bosque mixto de pino-encino y bosque de oyamel (Romero 2005). De acuerdo a la Lista Roja de Especies Amenazadas de la UICN, la especie se encuentra catalogada como de Baja Preocupación (Kelly et al. 2008) y como especie En Peligro por el U.S. Fish and Wildlife Service (1973). La pérdida de hábitat y la cacería ilegal son sus principales amenazas (Kelly et al. 2008).

En México se han realizado numerosos estudios sobre la ecología de esta especie, fundamentalmente con relación a su dieta (Romero 1987, Aranda et al. 2002, Bárcenas 2010). Sin embargo, actualmente se desconoce la viabilidad y tamaño de sus poblacionales, así como el estado de salud de las mismas, por lo que es necesario generar esta información para definir el estado actual de la especie en México. En este trabajo reportamos los primeros registros para México de Toxascaris leonina y Cystoisospora felis en una población silvestre de Lince Rojo en la Sierra del Ajusco-Chichinauhtzin, Distrito Federal y en el volcán Tláloc, Estado de México.

Los registros provienen del análisis de excretas recolectadas en la Sierra del Ajusco-Chichinauhtzin, Distrito Federal, en el 2013 (Gallardo-Santis 2014) y en el volcán Tláloc, Estado de México, en el 2014. Las muestras fueron colectadas en recorridos ad-libitum sobre senderos pre-establecidos, y fueron conservadas en formol al $4 \%$ para luego ser procesadas en el Laboratorio de Diagnóstico Parasitológico de la Facultad de Medicina Veterinaria y Zootecnia de la Universidad Nacional Autónoma de México. A través de la técnica de flotación con solución saturada de cloruro de sodio (Foreyt 2001, Besné et al. 2006, Bowman 2014), se identificaron los huevos de helmintos, así como los quistes y ooquistes de protozoarios. La identificación taxonómica se realizó a través de las claves de Levine (1985), Zajac \& Conboy (2012) y Bowman (2014).

En total se recolectaron y analizaron 258 excretas de Lince Rojo en la Sierra del Ajusco-Chichinauhtzin, con 25\% de las muestras positivas y 21 excretas en el volcán Tláloc, de las cuales el 14\% resultaron positivas. Las especies identificadas fueron el nematodo Toxascaris leonina (60 muestras Sierra del Ajusco-Chichinauhtzin, 2 muestras Volcán Tláloc, Figura 1A), y el protozoario Cystoisospora felis (5 muestras Sierra del Ajusco-Chichinauhtzin, 1 muestra volcán Tláloc, Figura 1B).

Mammalogy Notes | Notas Mastozoológicas

Sociedad Colombiana de Mastozoología

Vol. 2 Num. 1 | 2015 


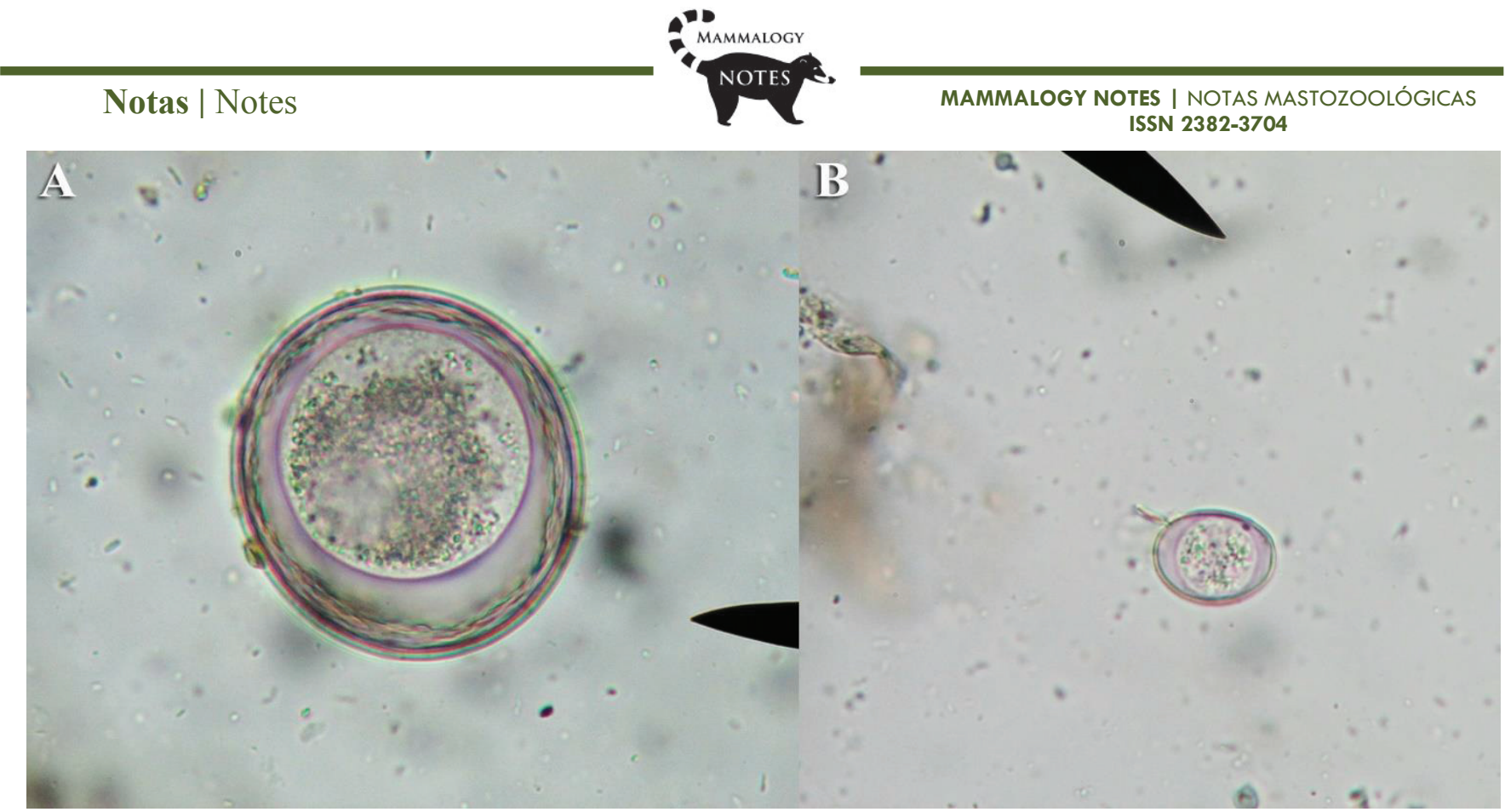

Figura 1. Fotografías microscópicas de (A) un huevo de Toxascaris leonina y un (B) ooquiste no esporulado de Cystoisospora felis con objetivo seco fuerte (40x).

Toxascaris leonina es una especie encontrada con frecuencia en cánidos y félidos (Bowman 2014), la larva infectante de esta especie se puede enquistar en los tejidos de hospederos paraténicos como roedores y seres humanos (Toxascariasis larval) por lo que se le considera una especie zoonótica (Bowman 2014). Cystoisospora felis es una especie que se encuentra principalmente en felinos, y que puede afectar el desarrollo y sobrevivencia de animales jóvenes, debido a los cuadros de diarreas agudas a crónicas característicos de esta parasitosis (Pérez-Tort et al. 2009, Bowman 2014). Estas dos especies han sido reportadas en poblaciones de Lynx rufus en diversos estudios parasitológicos realizados en Estados Unidos (Rollings 1945, Leiby 1961, Little et al. 1971, Stone \& Pence 1978, Schitoskey \& Linder 1981, Watson et al. 1981, Tiekotter 1985, Carver et al. 2012, Hiestand et al. 2014). Sin embargo, en México estudios parasitológicos en la especie han reportado únicamente la presencia de Toxoplasma gondii (Kikuchi et al. 2004) y Echinococcus oligarthrus en Tamaulipas (SalinasLópez 1996). En este sentido, los datos aquí encontrados representan los primeros registros de Toxascaris leonina y Cystoisospora felis en Lince Rojo para el país.

La información aquí presentada evidencia la necesidad de contar con una línea base sobre la salud y la diversidad de parásitos presentes en el Lince Rojo, y otros carnívoros silvestres, principalmente en los paisajes dominados por las actividades humanas. A su vez, resalta la necesidad de realizar proyectos a largo plazo para entender la ecología de la dinámica parásitohospedero y diseñar estrategias de conservación que permitan la sobrevivencia de las poblaciones de esta especie a largo plazo en el centro del México.

\section{Agradecimientos}

Agradecemos a la Universidad Autónoma del Estado de México por financiar parte de este estudio a través del proyecto (3530/2013MT), a la Universidad Nacional Autónoma de México por sus facilidades prestadas y a la Universidad Autónoma Metropolitana Unidad Lerma por apoyar el proyecto de investigación "Ecología y conservación de carnívoros en ambientes modificados por el hombre." Especial agradecimiento por sus comentarios y sugerencias a los editores que ayudaron a mejorar el manuscrito.

\section{Referencias}

ARANDA, M. et al. 2002. Análisis comparativo de la alimentación del gato montés (Lynx rufus) en dos diferentes ambientes de México. Acta Zoológica Mexicana 87: 99-109. BÁRCENAS, H. 2010. Abundancia y dieta del lince (Lynx rufus) en seis localidades de México. Tesis de Maestría en Ciencias Biológicas (Biología Ambiental), Instituto de Ecología, Universidad Nacional Autónoma de México.

BESNÉ, A. et al. 2006. Manual de prácticas de laboratorio de parasitología. México, Departamento de Parasitología. Facultad de Medicina Veterinaria y Zootecnia, UNAM. BOWMAN, D.D. 2014. Georgis' Parasitology for veterinarians. 10a edición. E.U.A., Elsevier Saunders.

CARVER, S. et al. 2012. Zoonotic parasites of bobcats around human landscapes. Journal of Clinical Microbiology 50: $3080-3083$.

Mammalogy Notes | Notas Mastozoológicas

Sociedad Colombiana de Mastozoología

Vol. 2 Num. 1 | 2015 
FOREYT, W.J. 2001. Veterinary parasitology. Reference Manual. 5a edición. Blackwell Publishing. E.U.A., Iowa State University Press.

GALLARDO-SANTIS, A. 2014. Endoparásitos en heces de linces, coyotes y perros que habitan un paisaje fragmentado del Ajusco, Distrito Federal, México. Tesis de Licenciatura en Medicina Veterinaria y Zootecnia, Facultad de Medicina Veterinaria y Zootecnia, Universidad Nacional Autónoma de México.

HALL, E.R. 1981. The mammals of North America. Volumen 2. E.U.A., Wiley.

HIESTAND, S.J. et al. 2014. Epizootic and zoonotic helminths of the bobcat (Lynx rufus) in Illiois and comparison of its helminth component communities across the American Midwest. Parasite 21: 1-9.

KELLY, M. et al. 2008. Lynx rufus. The IUCN Red List of Threatened Species. Version 2012.2. <www.iucnredlist.org>. Descargado el 30 de marzo de 2015.

KIKUCHI, Y. et al. 2004. Seroprevalence of Toxoplasma gondii in American free-ranging or captive pumas (Felis concolor) and bobcats (Lynx rufus). Veterinary Parasitology 120: $1-9$.

LEIBY, P.D. 1961. Intestinal helminths of some Colorado mammals. Journal of Parasitology 47: 311.

LEVINE, N.D. 1985. Veterinary Protozoology. E.U.A., Iowa University Press.

LITTLE, J.W. 1971. Incidence and geographic distribution of some nematodes in Texas bobcats. Texas Journal of Science 22: $302-314$.

PÉREZ-TORT, G. et al. 2009. Primera descripción del uso del Toltrazuril para la prevención de brotes de coccidiosis en criaderos felinos. Veterinaria Argentina XXVI: 252. http://www.veterinariargentina.com/revista/2009/04/719/

ROLLINGS, C.T. 1945. Habits, foods and parasites of the Bobcat in Minnesota. The Journal of Wildlife Management 9: 131-145.

ROMERO, F. 1987. Análisis de la alimentación del lince (Lynx rufus escuinapae) en el Volcán Pelado, Ajusco, Distrito Federal, México. Tesis Licenciatura en Biología, Facultad de Ciencias, Universidad Nacional Autónoma de México.

ROMERO, F. 2005. Lynx rufus (Schreber, 1777) Lince, gato montés. En: Los mamíferos silvestres de México (Ceballos, G. \& G. Oliva). México, CONABIO, Fondo de Cultura Económica.

SALINAS-LÓPEZ, N. et al. 1996. Presence of Echinococcus oligarthrus (Diesing, 1863) Liihe, 1910 in Lynx rufus texensis Allen, 1895 from San Fernando, Tamaulipas State, in North-East Mexico. International Journal of Parasitology 26: 793-796

SCHITOSKEY, E. C. \& R. L. Linder. 1981. Helminths of South Dakota bobcats. Proc. S.D. Acad. Sci. 60: 135-141.

STONE, J.E. \& D.B. PENCE. 1978. Ecology of helminth parasitism in the bobcat from west Texas. Journal of Parasitology 64: $295-302$.

TIEKOTTER, K.L. 1985. Helminth Species Diversity and Biology in the Bobcat, Lynx rufus (Schreber), from Nebraska. Journal of Parasitology 71 : $227-234$.

U.S. FISH \& WILDLIFE SERVICE. 1973. Endangered Species Act. Mexican Bobcat. Endangered Species Program. US.

WATSON, T.G. et al. 1981. Endoparasites and selected infectious agents in bobcats (Felis rufus), from West Virginia and Georgia. Journal of Wildlife Diseases 17: 547-554.

ZAJAC, A.M. \& G.A. CONBOY. 2012. Veterinary clinical parasitology. 8a edición. E.U.A, Wiley-Blackwell. 